\title{
RECENT RESULTS FROM THE TESLA TEST FACILITY (TTF)
}

\section{Hüning*, RWTH Aachen and Deutsches Elektronen Synchrotron DESY, Germany for the TESLA Collaboration}

\begin{abstract}
In this paper we present recent results of the TESLA Test Facility (TTF). The most important milestone was the operation with the TESLA design current, macropulses of $800 \mu$ s with bunches of $4 \mathrm{nC}$ at a rate of $2.25 \mathrm{MHz}$.

Further studies include measurements of higher order modes (HOMs) in superconducting cavities, optimization of the TTF Free Electron Laser at $108 \mathrm{~nm}$, an experiment on wakefields in rough beam pipes, and new developments for sub-picosecond bunch length measurements.

Latest cavity test results show a reliable fulfillment of the TESLA goal of $23.5 \mathrm{MV} / \mathrm{m}$.
\end{abstract}

\section{INTRODUCTION}

The TESLA Test Facility (TTF) includes the infrastructure for chemical treatment and clean-room assembly of industrially produced 9 -cell cavities. To date, more than sixty 9-cell cavities have been processed and tested. The TTF linac (TTFL) at DESY was constructed to demonstrate that the high gradients achieved in the cavities could be maintained during assembly into a linac test string, and that cavities and auxiliary systems can be successfully operated to accelerate an electron beam to several hundred $\mathrm{MeV}$. The basic characteristics of the TTFL were designed to be as similar as possible to the parameters of the TESLA collider.

The TTFL is equipped with a $13.5 \mathrm{~m}$ long undulator magnet for generating FEL radiation according to the principle of Self Amplified Spontaneous Emission (SASE). Meanwhile the SASE FEL has been successfully demonstrated at wavelengths between 83 and $180 \mathrm{~nm}$ [2].

\section{CAVITY TESTING}

The cavities for TTF are fabricated by industry. Niobium sheets are deep drawn to half cells which are then electron-beam welded to 9-cell cavity structures. The standard treatment of the cavities includes annealing at $800^{\circ} \mathrm{C}$, a heat treatment at $1400^{\circ} \mathrm{C}$ with titanium getter, several steps of Buffered Chemical Polishing (BCP), and three steps of high-pressure water rinsing (100 bar). The performance test of the cavities is carried out in a vertical bath cryostat in cw operation. Figure 1 shows a summary of the test results. The depicted gradients were achieved with a $Q_{0}>10^{10}$. The most recent cavities fulfill the TESLA goal of $23.5 \mathrm{MV} / \mathrm{m}$ (for $500 \mathrm{GeV} \mathrm{CM}$ energy) with a comfortable safety margin. Tests are ongoing to replace the $\mathrm{BCP}$ by Electro Polishing (EP) and to avoid the heat treatment at $1400^{\circ} \mathrm{C}$. The results are very promising, gradients

\footnotetext{
*markus.huening@desy.de
}
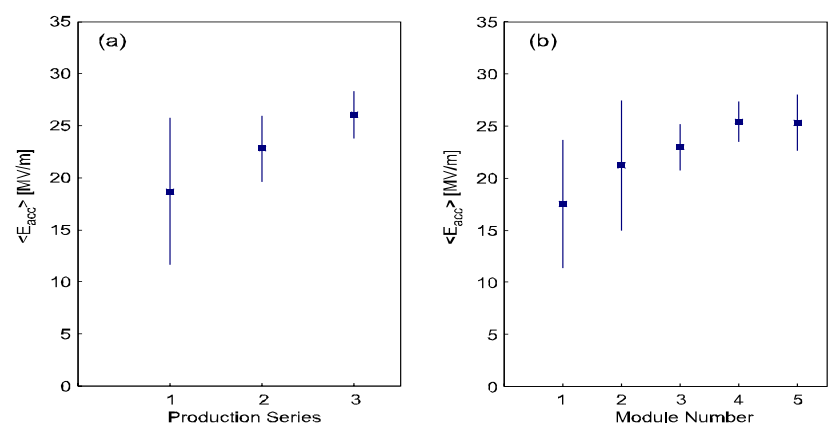

Figure 1: Left: average performance of the cavities of three production series. The depicted gradients are the mean values of the gradients achieved at $Q_{0}=10^{10}$ for cavities without known material or fabrication defects. The error bars show the rms value of the distribution. Right: average gradient of the cavities installed in the first 5 cryomodules (each containing 8 cavities).

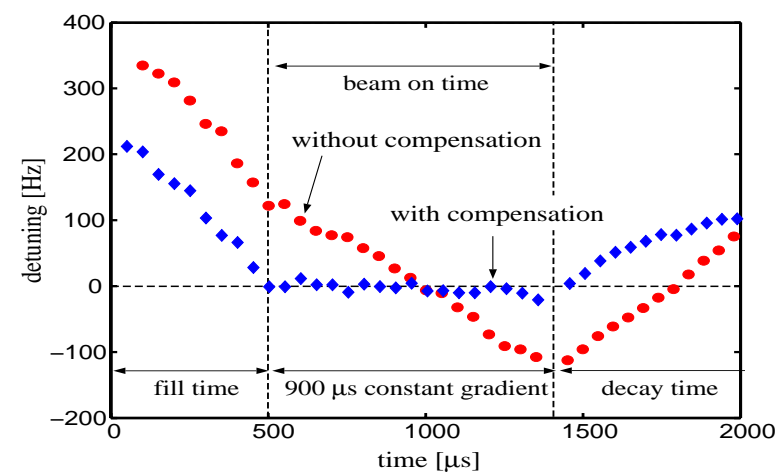

Figure 2: Compensation of Lorentz-force detuning using a piezo tuner.

between 35 and $42 \mathrm{MV} / \mathrm{m}$ are routinely being achieved with single-cell cavities [1].

For installation into the cryostats the cavities are welded into helium tanks and equipped with high power couplers. They are tested in a horizontal cryostat in pulsed mode to simulate the operation in the accelerator. In pulsed mode the cavities exhibit dynamic Lorentz-force detuning: the resonance frequency shifts quadratically with the accelerating field. The cavities are stiffened to reduce the detuning, but at gradients of $25 \mathrm{MV} / \mathrm{m}$ still $20 \%$ extra rf power is needed to achieve the required field stability. Lorentz-force detuning has been successfully compensated by means of a piezo tuner which is fast enough to correct the detuning of the cavity during the rf pulse. Test results are shown in figure 2. 


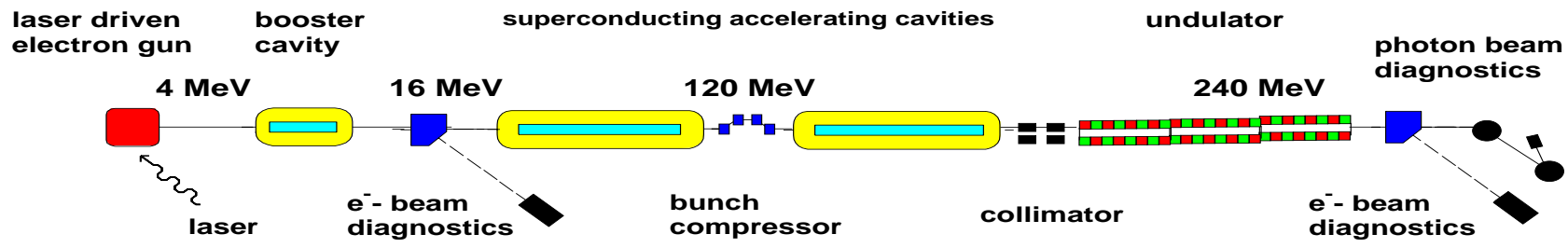

Figure 3: Scetch of the TTF linac in its present state of development

\section{TESLA BEAM PARAMETERS}

The design of TESLA foresees a bunch charge of $3.2 \mathrm{nC}$ with a bunch spacing of $337 \mathrm{~ns}$ in macro pulses of $800 \mu \mathrm{s}$ length with a repetition rate of $5 \mathrm{~Hz}$. At TTFL it has been shown that a beam of this pulsestructure can be accelerated. The achieved energy stability was well within the requirements (see figure 4).

A fast interlock system switches off the photocathode laser whenever beam losses exceed a specific threshold. The requirements for the stability are very tight, because the undulator for the FEL is made from permanent magnets which are vulnerable to radiation damage (tolerable dose $<10^{5} \mathrm{~Gy}$ ). The interlock system consists of two parts: the beam transport along the linac is monitored with toroids, and the losses are recorded with scintillator counters at sensitive points of the machine. In case of too high losses the beam is switched off within $3 \mu$ s, i.e. after 10 bunches.

\section{HIGHER ORDER MODES}

Higher order modes (HOM) in the accelerating structures are a major concern for all linear collider designs. In the TTFL an experiment has been conducted to measure beam induced dipole HOMs [3]. For this purpose the bunch repetition rate was raised to $54 \mathrm{MHz}$. The beam was passed off-axis through the first acceleration module. The beam current was modulated with a variable frequency between 0 and $27 \mathrm{MHz}$. Whenever one of the sidebands of the modulation hits a HOM frequency a coherent excitation is

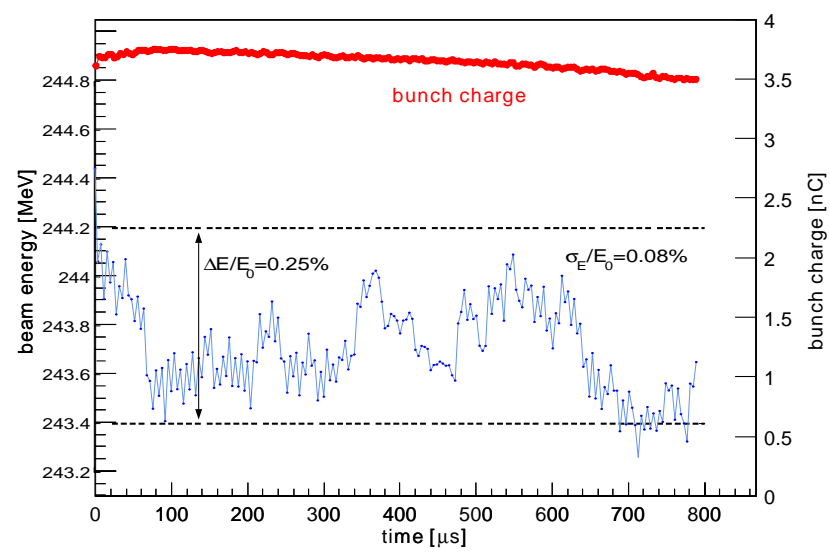

Figure 4: Energy and charge stability achieved with long macropulses.

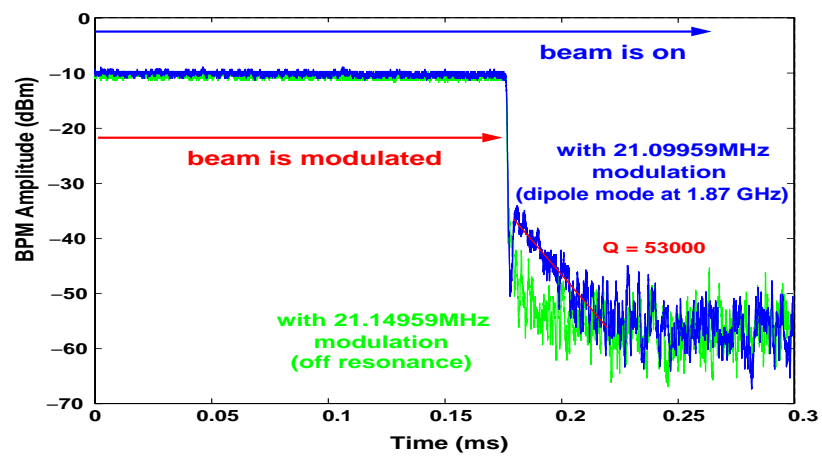

Figure 5: Detection of deflecting higher order modes. After switching off the amplitude modulation of the beam current (here $21.09959 \mathrm{MHz}$ ) a modulation of the BPM signal is caused by a exponentially decaying HOM, off resonance the signal decays much faster.

expected. If a dipole mode or higher multipole is excited the beam position at a downstream beam position monitor (BPM) will be modulated with the same frequency. To measure the deflection a wideband BPM was used. The variable filter of a spectrum analyzer was used to select the expected frequency according to the modulation chosen. To avoid parasitic effects the deflection was measured after switching off the amplitude modulation. The unmodulated beam then probes the field decay of the HOM (see figure 5).

The location of the HOM can be found by successively detuning the cavities. The frequency of the mode is measured at the HOM-couplers of the cavity. The figure 6 shows the result of an integral scan. Many of the strongest excitations have been preliminarily attributed to quadrupole modes which can be neglected for the small beam offsets during normal operation. In the experiment they are artificially enhanced due to the large offset of $20 \mathrm{~mm}$. For further details see [3].

\section{SURFACE ROUGHNESS WAKEFIELDS}

One of the most important contributions to the performance of a SASE FEL are the wakefield effects inside the undulator. Simulations gave hints that surface roughness plays a significant role [6][7]. Several models were developed with rather different predictions. Therefore an experiment was designed to test the model calculations. In a section of $80 \mathrm{~cm}$ length a series of beam pipes with various 


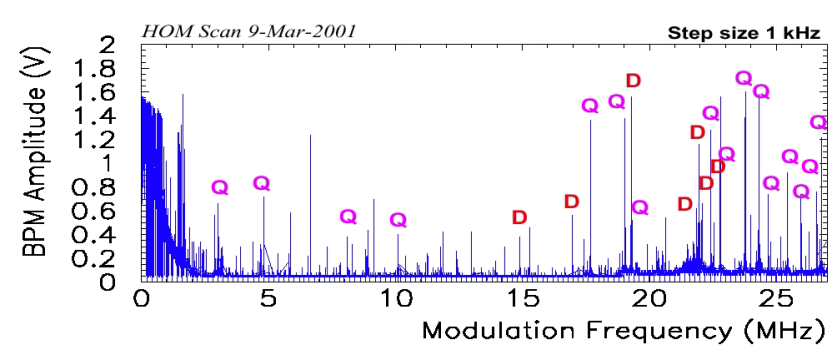

Figure 6: Total scan of the excitation frequencies. Plotted are the maximum amplitudes of the deflection after switching off the modulation. The 20 candidates with the strongest deflection were studied more closely. The asignment to quadrupole $(\mathrm{Q})$ and dipole $(\mathrm{D})$ modes is preliminary.
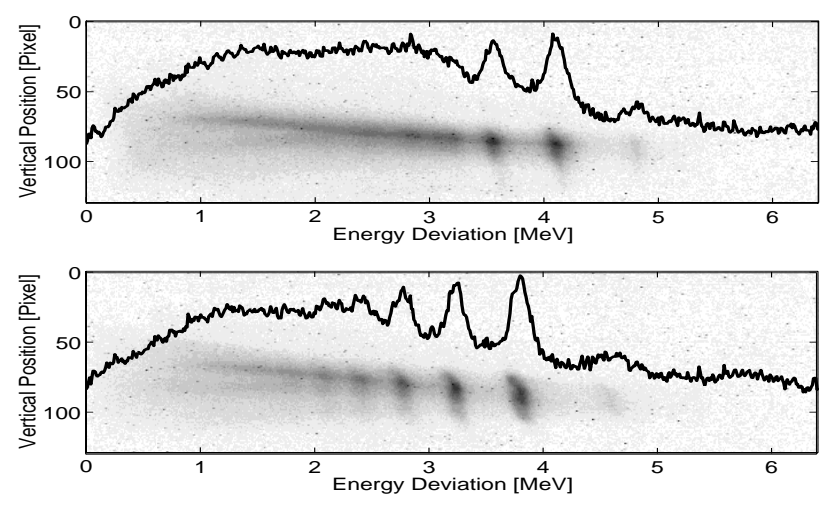

Figure 7: Change of the energy distribution of the bunches due to the wakefields excited by a rough surface of the beam pipe. The upper part shows the distribution with a smooth beam pipe, the lower one after insertion of a roughened pipe. The result is in agreement with a harmonic wake potential as predicted by the dielectric layer model[6]. The structure in the upper graph is probably caused by wakefields generated upstream.

surface treatments were installed.

The figure 7 shows the effect on the energy distribution of the bunch. The roughened beam pipe generates a substructure in the bunch which can be explained by a harmonic wake potential as predicted by the dielectric layer model. Independently the emission of THz-radiation has been observed. The measured frequencies around $500 \mathrm{GHz}$ fit well with the expectation from the model. Detailed analysis is ongoing.

\section{BEAM BASED ALIGNMENT}

At the Technical University of Darmstadt a computer code has been developed to simulate space charge dominated high current beams as they are produced in the rf photo cathode of the TTFL [4]. Based on these simulations a beam based alignment procedure was carried out to center the solenoids surrounding the rf gun. Already the first iteration delivered good results (see figure 8).

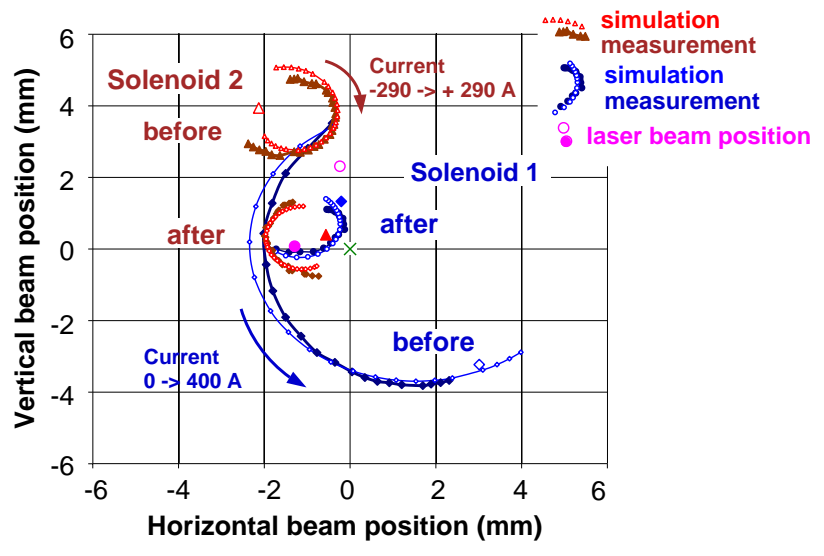

Figure 8: First iteration of beam based alignment of the rf gun solenoids. An online simulation program was used to fit measured beam positions as function of solenoid current. The resulting values for the solenoid offsets were used to correct the alignment.

\section{CONCLUSION AND OUTLOOK}

The cavity production and processing has reached a state of maturity where the TESLA $(500 \mathrm{GeV})$ specification of $23.5 \mathrm{MV} / \mathrm{m}$ is fulfilled with an adequate safety margin. Lorentz-force detuning can be compensated by a piezo tuner. The acceleration of long macropulses with the design bunch charge has been demonstrated.

Beam-induced HOMs were experimentally studied. Surface roughness wakefields have been studied experimentally. The preliminary results agree with the predictions of the dielectric layer model. A code to simulate space charge dominated beams in rf guns has been successfully applied to align the solenoid coils of the gun.

\section{REFERENCES}

[1] J. Andruszkow et al., TESLA Technical Design Report, Part II: The Accelerator, DESY 2001 - 011, ECFA 2001 - 209, TESLA Report 2001 - 23, TESLA - FEL 2001 - 05

[2] J. Andruszkow et al., First Observation of Self-Amplified Spontaneous Emission in a Free-Electron Laser at $109 \mathrm{~nm}$ Wavelength, Phys. Rev. Letters Vol. 85 (2000), No. 18 pp. 3825-3829

[3] N. Baboi et al., Measurements with Beam of the Deflecting Higher Order Modes in the TTF Superconducting Cavities, This Proceedings ID 2197 This Proceedings ID 1771

[4] M. Krassilnikov, Beam Based Alignment of the TTF RF-Gun using V-Code, This Proceedings ID 1102

[5] M. Liepe, W.D. Möller, S.N. Simrock, Dynamic LorentzForce Compensation with a Fast Piezoelectric Tuner, This Proceedings ID 2078

[6] A. Novokhatski, M. Timm, T. Weiland, The surface Roughness Wakefield Effect, EPAC 98, Stockholm, May 1998

[7] R.E. Stupakov, D. Thomson, R.C. Walz, Effects of beam tube roughness on $X$-ray free electron laser performance, Phys. Rev. ST Accel. Beams 2 (6) (1999) 060701 